\title{
INFRARED STUDIES OF EPSILON AURIGAE IN ECLIPSE
}

\author{
Robert E. Stencel ${ }^{1}$, Brian K. Kloppenborg ${ }^{1}$, Randall E. Wall, Jr. ${ }^{1}$, Jeffrey L. Hopkins ${ }^{2}$, Steve B. Howell $^{3}$, \\ D. W. Hoard ${ }^{4}$, John Rayner ${ }^{5}$, Schelte Bus $^{5}$, Alan Tokunaga ${ }^{5}$, Michael L. Sitko ${ }^{6,7}$, Suellen Bradford $^{6,7}$, \\ Ray W. Russell ${ }^{8}$, David K. LynCH $^{8}$, Heidi Hammel ${ }^{9}$, Barbara Whitney ${ }^{9}$, Glenn Orton ${ }^{10}$, \\ Padma Yanamandra-Fisher ${ }^{10}$, Joseph L. Hora ${ }^{11}$, Philip Hinz ${ }^{12}$, William Hoffmann ${ }^{12}$, AND ANDREW SKemeR ${ }^{12}$ \\ ${ }^{1}$ Department of Physics \& Astronomy, University of Denver, Denver, CO 80208 USA; rstencel@du.edu \\ ${ }^{2}$ Hopkins Phoenix Observatory, Phoenix, AZ 85033 USA \\ ${ }^{3}$ National Optical Astronomy Observatories, Tucson, AZ 85719, USA \\ ${ }^{4}$ Spitzer Science Center, California Institute of Technology, Pasadena, CA 91125, USA \\ ${ }^{5}$ Institute for Astronomy, University of Hawaii, Honolulu, HI 96822, USA \\ ${ }^{6}$ Department of Physics, Cincinnati University, Cincinnati, OH, USA \\ ${ }^{7}$ Space Science Institute, Boulder, CO, USA \\ ${ }^{8}$ The Aerospace Corporation, Los Angeles, CA 90009, USA \\ ${ }^{9}$ Space Science Institute, Boulder, CO 80301, USA \\ 10 Jet Propulsion Laboratory, California Institute of Technology, Pasadena, CA 91109, USA \\ ${ }^{11}$ Harvard-Smithsonian Center for Astrophysics, Cambridge, MA 02138, USA \\ ${ }^{12}$ Steward Observatory, Department of Astronomy, University of Arizona, Tucson, AZ 85721, USA \\ Received 2011 July 7; accepted 2011 September 16; published 2011 October 17
}

\begin{abstract}
We report here on a series of medium resolution spectro-photometric observations of the enigmatic long period eclipsing binary epsilon Aurigae, during its eclipse interval of 2009-2011, using near-infrared spectra obtained with SpeX on the Infrared Telescope Facility (IRTF), mid-infrared spectra obtained with BASS on AOES and IRTF, MIRSI on IRTF, and MIRAC4 on the MMT, along with mid-infrared photometry using MIRSI on IRTF and MIRAC4 on the MMT, plus 1995-2000 timeframe published photometry and data obtained with Denver's TNTCAM2 at WIRO. The goals of these observations included: (1) comparing eclipse depths with prior eclipse data, (2) confirming the re-appearance of $\mathrm{CO}$ absorption bands at and after mid-eclipse, associated with sublimation in the disk, (3) seeking evidence for any mid-infrared solid state spectral features from particles in the disk, and (4) providing evidence that the externally irradiated disk has azimuthal temperature differences. IR eclipse depths appear similar to those observed during the most recent (1983) eclipse, although evidence for post-mid-eclipse disk temperature increase is present, due to $\mathrm{F}$ star heated portions of the disk coming into view. Molecular $\mathrm{CO}$ absorption returned 57 days after nominal mid-eclipse, but was not detected at mid-eclipse plus 34 days, narrowing the association with differentially heated sub-regions in the disk. Transient He I 10830A absorption was detected at mid-eclipse, persisting for at least 90 days thereafter, providing a diagnostic for the hot central region. The lack of solid-state features in Spitzer Infrared Spectrograph, BASS, and MIRAC spectra to date suggests the dominance of large particles (micron-sized) in the disk. Based on these observations, mid-infrared studies out of eclipse can directly monitor and map the disk thermal changes, and better constrain disk opacity and thermal conductivity.
\end{abstract}

Key words: binaries: eclipsing - protoplanetary disks - stars: individual (epsilon Aurigae)

Online-only material: color figures

\section{INTRODUCTION}

The bright eclipsing binary star, $\epsilon$ Aurigae, has long fascinated astronomers because they could determine the nature of one star in the system, but not the other (for a review, see Guinan $\&$ Dewarf 2002). The crux of the problem was that the early F type star in this single-lined spectroscopic binary resembles a supergiant and thus should have a comparably massive companion $\left(\sim 10-15 M_{\odot}\right)$. However, the companion is vastly underluminous for the inferred mass. Various models have been proposed, but one model involves a post-asymptotic giant branch F star and a fairly normal upper main-sequence star surrounded by a disk of obscuring material (Huang 1965; Lambert \& Sawyer 1986). Evidence for the existence of this disk was first provided by Backman et al. $(1984,1985)$, who detected the infrared presence of an extended, $500 \mathrm{~K}$ source in the system. Predictions of azimuthal asymmetry in disk structure, due to external heating by the $\mathrm{F}$ star, have been provided by Takeuti $(1986,2011)$. Interferometric $H$-band imaging during the start of the current eclipse has confirmed the existence of the disk and provided strong constraints on its dimensions and the system mass ratio (Kloppenborg et al. 2010, hereafter KSM). These considerations and more set the stage for the confirmation and extension of prior results, given this first eclipse of the 21st century (see Stencel 2010).

\subsection{IR Light Curves}

We are fortunate to have well-determined optical light curves, due to the International Campaign organized by Jeffrey Hopkins (Hopkins \& Stencel 2011), which has provided thousands of UBVRIJH measurements since 2006 and earlier. From these data, times of contact have been established as: First $=$ RJD 55,060 ( $V \sim 3.00)$; Second $=$ RJD 55,200 $(V \sim 3.80)$; mid-eclipse circa RJD 55,390 \pm 10 days $(V \sim 3.75)$. RJD = JD minus 2,400,000. Here we denote Second and Third contacts as reference points, even though the non-circular disk causing the eclipse will not produce a sharp second or third contact in the traditional eclipsing binary star sense. Observed times of egress were Third $=$ RJD 55,620 ( $V \sim 3.80)$ and Fourth $=$ RJD 55,720 $(V \sim 3.1)$. Note that egress also featured a pronounced change 
of slope starting at RJD 55,360 ( $V \sim 3.3)$. The persistence of out of eclipse variations during totality adds some uncertainty as well, but we adopt the foregoing times of contact for use in the discussion that follows, anticipating that small refinements will be forthcoming, as part of post-eclipse analyses.

A re-analysis of the spectral energy distribution (SED) by Hoard et al. (2010, hereafter HHS) shows a transition between the $\mathrm{F}$ star $(7500 \mathrm{~K})$ and the cold disk $(550 \pm 50 \mathrm{~K}$ during total eclipse phase) at/near $3 \mu \mathrm{m}$. The combined effects of eclipse attenuation of the $\mathrm{F}$ star and the predicted temperature rise as heated portions of the disk come into view after mideclipse (Takeuti 1986, 2011); both should result in measurable changes to the SED at near- and mid-IR wavelengths. The first effect was exploited by Backman et al. (1984) during the last eclipse, and they thus discovered the $550 \mathrm{~K}$ disk signature, using 2-20 $\mu \mathrm{m}$ wavelength-dependent eclipse depths, with Capella as the primary calibration star. However, their published monitoring only covered the first-half of the long eclipse, and did not address the second effect, the externally heated disk that comes increasingly into view after mid-eclipse. In this paper, we seek to measure and quantify both effects.

\subsection{Spectral Line Changes: $\mathrm{CO}$ and He I}

During the previous eclipse in 1983, Hinkle \& Simon (1987) collected high resolution, $K$-band FTS data that showed a strong asymmetry in the strength of molecular CO (2-0) spectral features in the near-infrared $(2.29 \mu \mathrm{m})$, appearing only after mid-eclipse, and persisting well past optical fourth contact (the nominal end of eclipse). They described the $\mathrm{CO}$ column density as being at least 80 times larger in the trailing edge of the disk compared to the leading edge, with excitation temperatures rising to $1000 \pm 150 \mathrm{~K}$, and column densities reaching $3 \times$ $10^{20} \mathrm{CO} \mathrm{cm}{ }^{-2}$. From velocity measurements and for Keplerian rotation, they constrained component masses to be 4-7 $M_{\odot}$ for the F star, and 7-10 $M_{\odot}$ for the disk and its central object. This is consistent with the 2:3 mass ratio deduced interferometrically (KSM). It is also comparable to the $6 M_{\odot}$ central star of the disk, inferred from the SED (HHS), as well as the 3-6 $M_{\odot}$ for the central star inside the disk, and less than $3 M_{\odot}$ for the $\mathrm{F}$ star, deduced by Lambert \& Sawyer (1986) based on velocities derived from the optically thin K I 7699A line. Hinkle and Simon also reported a large enhancement in the ${ }^{13} \mathrm{C}$ abundance in the disk gas, indicating that nuclear processing has occurred in the assumed source, the F star, implying that it is an evolved object. In this paper, we confirm the re-appearance of $\mathrm{CO}$ again after mid-eclipse in 2010. Also, as a result of this monitoring, the remarkable He I 10830A line was detected for the first time, appearing strongly in absorption following mid-eclipse (atop a weak photospheric feature previously and subsequently seen). Details are given below.

\subsection{Limits on Solid State Features}

Less well constrained is the dust-to-gas ratio of the disk and its overall mass, in part because there are no dust signatures detected. The infrared spectrum provided by the Spitzer Infrared Spectrograph (IRS) presents a largely featureless continuum, implying large particles $(\geqslant 0.1 \mu \mathrm{m})$. The analogy with the appearance of $\mathrm{CO}$ suggests the possibility that $10 \mu \mathrm{m}$ silicates, due to smaller particles, may appear after mid-eclipse due to sublimation by $\mathrm{F}$ star heating. In this paper, we report on a series of mid-infrared photometric and spectroscopic observations to investigate this. Defining the dust-to-gas ratio remains an important goal in the study of this disk and relating it to other transitional disks.

\section{THE OBSERVATIONS}

Our observational objectives included monitoring infrared flux levels at multiple wavelengths, exploring spectral line profile changes, and especially watching for the predicted re-appearance of molecular $\mathrm{CO}(2-0)$ bands near $2.3 \mu \mathrm{m}$. Observations upon which this paper is based were obtained with a variety of telescopes and instruments, as detailed below. Photometric and spectroscopic observations were obtained at irregular intervals depending on time allocations, weather, and other factors. Optical photometry, spectroscopy, spectropolarimetry, and interferometry were pursued in parallel as part of the overall eclipse campaign, and those results are reported elsewhere.

\subsection{The Near-IR: IRTF/SpeX}

SpeX is a moderate resolution $(R \sim 2500)$, near-IR $(1-5 \mu \mathrm{m})$ spectrometer located at the NASA Infrared Telescope Facility (IRTF), as described by Rayner et al. (2003). Observations were made as early as 2008 and subsequently with increasing frequency, to as often as monthly during the second-half of the eclipse in 2010. Routine telluric correction and calibration were accomplished by comparison with simultaneous observations of nearby A0V stars (notably HD30169 and HD29526), using SpeXtool (Cushing et al. 2004; Vacca et al. 2003). Further calibration checks were made in comparison with broadband $J$ and $H$ measurements and slitless Broadband Array Spectrograph System (BASS) data (see below). In Table 1 we list the SpeX observational details, and in Table 2 we list the monochromatic fluxes in $\mathrm{W} \mathrm{m}^{-2}$ at selected continuum wavelengths. Individual signal-to-noise-based uncertainties formally were less than $1 \%$ at the shortest wavelengths, increasing toward longer wavelengths unless noted otherwise. Absolute calibration is estimated to lie within an uncertainty range of less than $5 \%$.

\subsubsection{Continuum Variations}

Figure 1 shows SpeX observations during eclipse ingress and totality phases. Corrections for telluric features in the spectra are not perfect, but the overall continuum is substantially decreased during the eclipse. During eclipse, Backman et al. (1984) noted uniform decrease at $J, H, K$, and $L$ bands $[1.25,1.65,2.2$, and $3.80 \mu \mathrm{m}$, respectively] of $0.72 \pm 0.03 \mathrm{mag}$, then decreasing at $M$ band $[4.8 \mu \mathrm{m}]$ to $0.66 \mathrm{mag}$, at $N$ band $[10.1 \mu \mathrm{m}]$ to $0.61 \mathrm{mag}$, and at $Q$ band [20 $\mu \mathrm{m}]$ to $0.32 \pm 0.06 \mathrm{mag}$. These correspond to flux ratios at JHKL of 0.52 , at $M$ of 0.55 , at $N$ of 0.57 , and at $Q$ of 0.74, which Backman et al. recognized as the spectral energy dominance of the cold disk affecting the longest wavelength eclipse depth ratios.

Our data appear consistent with these reported eclipse depth ratios. When we compare the monochromatic flux ratios between 2009 September 10 (first contact) and 2010 February 23 (second contact), we find 0.56 at $1.1 \mu \mathrm{m}, 0.55$ at $1.65 \mu \mathrm{m}, 0.54$ at $2.1 \mu \mathrm{m}, 0.51$ at $3.15 \mu \mathrm{m}, 0.43$ at $3.95 \mu \mathrm{m}$, but then rising to 0.55 at $4.64 \mu \mathrm{m}$. Our longest SpeX wavelengths are the most affected by telluric correction. For the shorter wavelengths, these appear consistent with Backman et al., within 3\% uncertainty. However, if we ratio fluxes between our pre-eclipse data (2008 January 27) and second contact (2010 February 24), we obtain deeper eclipse ratios: 0.45 at $1.1 \mu \mathrm{m}, 0.45$ at $1.65 \mu \mathrm{m}, 0.45$ at 


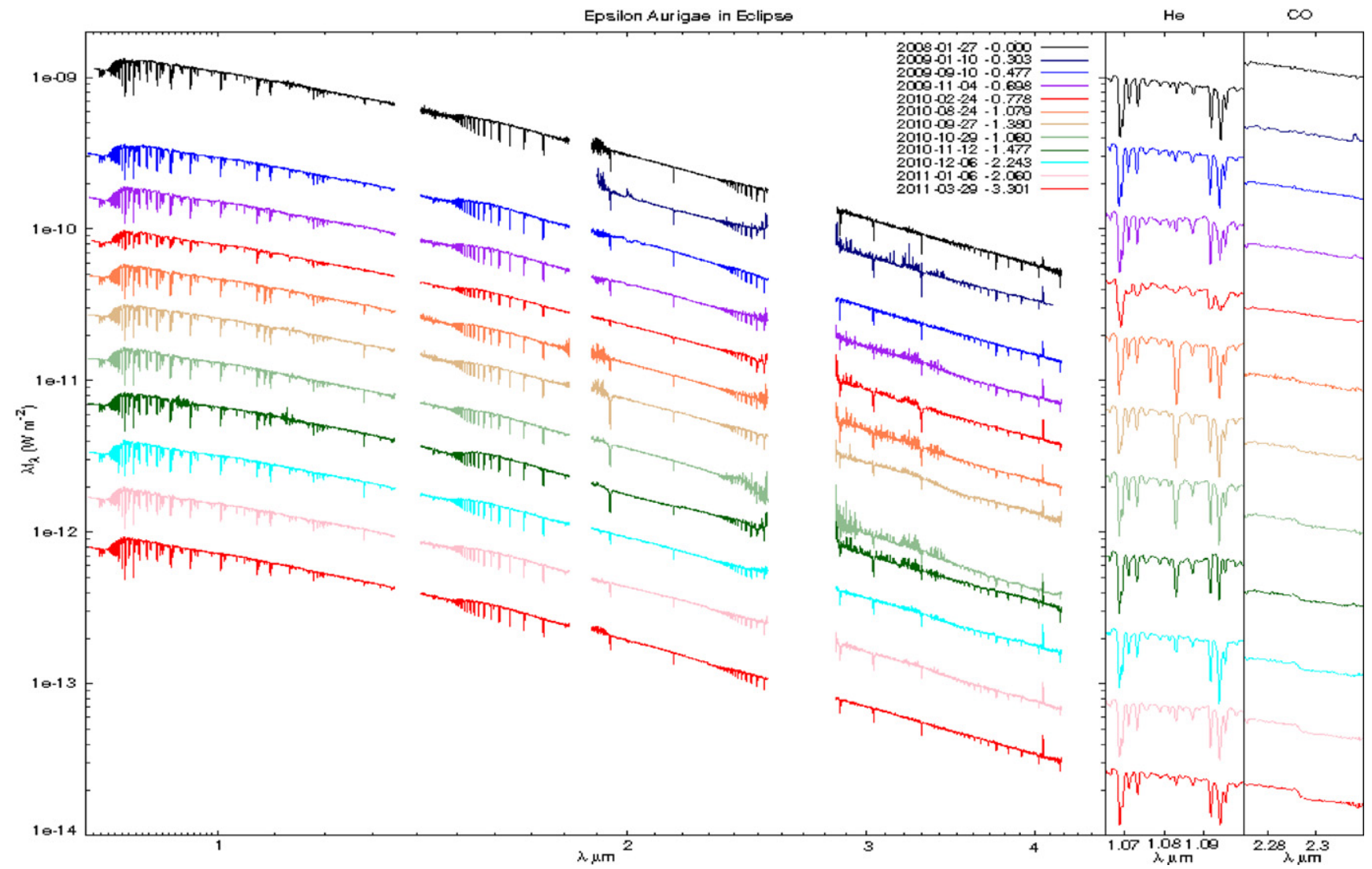

Figure 1. SpeX record of the eclipse of epsilon Aurigae, starting with pre-eclipse data in 2008, ingress (fall 2009), and totality (2010). These data reveal continuum and line changes, including transient He I ( $1.0830 \mu \mathrm{m}$, detailed in the first box, right) during mid-eclipse (2010 August-December), plus $2.29 \mu \mathrm{m}$ CO absorption appearing after mid-eclipse (second box, far right). For clarity, the continuum levels have been offset by the logarithmic factors next to the date caption list.

Table 1

SpeX Observations, SXD and LXD, 0.8-4.6 $\mu \mathrm{m}$

\begin{tabular}{|c|c|c|c|}
\hline JD & Date & Observers & Notes \\
\hline $2,454,493$ & 2008 Jan 27 & JTR et al. & $V=3.00$, slit $0.5, \mathrm{CO}$ absent \\
\hline $2,454,842$ & 2009 Jan 10 & RES/BKK & $V=2.99$, slit 0.3, CO absent, LXD only \\
\hline $2,455,084$ & 2009 Sep 10 & JTR et al. & $V=3.12$, slit $0.5, \mathrm{CO}$ absent \\
\hline $2,455,140$ & 2009 Nov 4 & JTR et al. & $V=3.43$, slit $0.5, \mathrm{CO}$ absent \\
\hline $2,455,167$ & $2009 \operatorname{Dec} 1$ & MS et al. & $V=3.53$, slit $0.8, \mathrm{CO}$ absent \\
\hline $2,455,250$ & $2010 \mathrm{Feb} 24$ & RES/BKK & $V=3.77$, slit $0.8, \mathrm{CO}$ absent \\
\hline $2,455,432$ & 2010 Aug 24 & RES/BKK & $V=3.73$, slit $0.3, \mathrm{CO}$ absent, He present \\
\hline $2,455,467$ & 2010 Sep 27 & RES/BKK & $V=3.69$, slit $0.3, \mathrm{CO}, \mathrm{He}$ present \\
\hline $2,455,494$ & 2010 Oct 24 & MS et al. & $V=3.72$, slit $0.8, \mathrm{CO}, \mathrm{He}$ present \\
\hline $2,455,499$ & 2010 Oct 29 & RES/BKK & $V=3.74$, slit $0.3, \mathrm{CO}, \mathrm{He}$ present \\
\hline $2,455,513$ & 2010 Nov 12 & RES/BKK & $V=3.76$, slit $0.3, \mathrm{CO}, \mathrm{He}$ present, cirrus \\
\hline $2,455,537$ & $2010 \operatorname{Dec} 6$ & RES/BKK & $V=3.72$, slit $0.3, \mathrm{CO}$ present \\
\hline $2,455,569$ & 2011 Jan 6 & RES/BKK & $V=3.76$, slit $0.3, \mathrm{CO}$ present \\
\hline $2,455,624$ & 2011 Mar 4 & MS et al. & $V=3.72$, slit $0.8, \mathrm{CO}$ present, clouds \\
\hline $2,455,649$ & 2011 Mar 29 & JTR et al. & $V=3.55$, slit $0.5, \mathrm{CO}$ present \\
\hline $2,455,829$ & 2011 Sep 24 & RES/BKK & $V=3.01$, slit $0.3, \mathrm{CO}$ absent \\
\hline
\end{tabular}

$2.1 \mu \mathrm{m}, 0.39$ at $3.15 \mu \mathrm{m}, 0.33$ at $3.95 \mu \mathrm{m}$, and 0.41 at $4.64 \mu \mathrm{m}$. Our pre-eclipse data were obtained 591 days before our first contact observation, when $V$ was slightly brighter than average. In the Backman et al. data, pre-eclipse IR magnitudes were obtained over a time span of 916 days $(J=1.77)$ to 263 days $(J=1.81)$ prior to the first contact, although simultaneous $V$ magnitudes were not available for comparison. We infer that effects of the cold disk material impact the infrared spectrum prior to optical first contact, consistent with neutral potassium 7699A equivalent width increases that were detected weeks prior to $V$-band first contact (Leadbeater \& Stencel 2010).
The $\sim 0.1$ mag optical photometric variations out of eclipse and during totality are well known, and are seen to persist during the 2010 eclipse. During autumn 2010, those variations were particularly well seen, with a local maxima at RJD 55467 and 55550 (Figure 2). The flux ratio of the local maximum at RJD 55647 to the local minimum that follows at RJD 55499 is 1.18 at $1.1 \mu \mathrm{m}, 1.24$ at $1.65 \mu \mathrm{m}, 1.27$ at $2.1 \mu \mathrm{m}, 1.22$ at $3.15 \mu \mathrm{m}, 1.29$ at $3.95 \mu \mathrm{m}$, and 1.39 at $4.65 \mu \mathrm{m}$. UBVRI magnitude changes reported by Hopkins et al. over this same interval include $\Delta U=0.10, \Delta B=0.09$ and $\Delta V=0.08, \Delta R=$ 0.06 and $\Delta I=0.02 \mathrm{mag}$. As small changes in magnitude are 

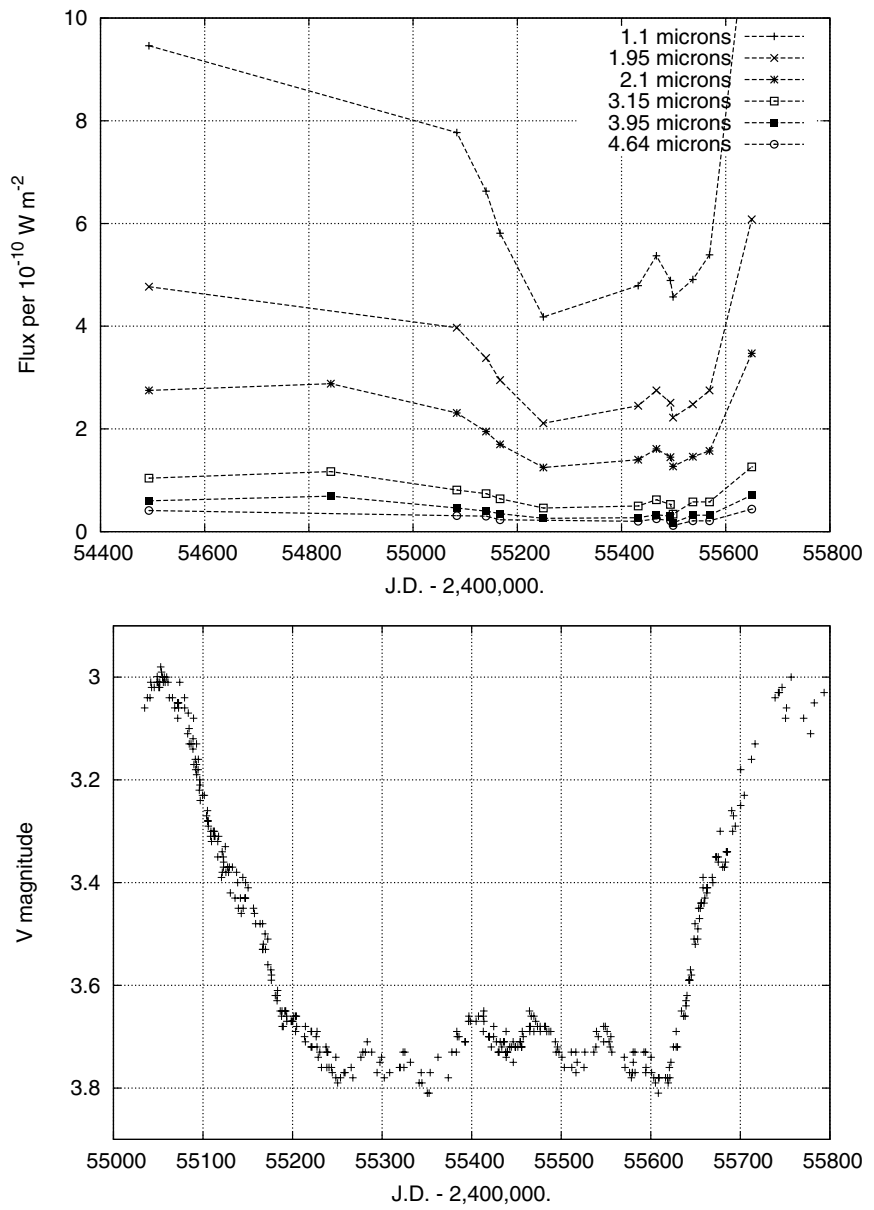

Figure 2. SpeX observations of the continuum differences at different wavelengths, between local optical light maxima [RJD 55,467 and 55,550] and local minima [55, 432 and 55, 499], compared with the $V$-band light curve (observer names include J. Hopkins, R. Miles, D. Loughney, C. Hofferber, H. G. Lindberg, Th. Karlsson, E. Guinan, M. Strikis, F. Melillo, and others-see Hopkins \& Stencel 2011).

close to ratio quantities, the flux variation seems to be minimum in the red, but larger in the near-UV and near-IR. The lack of any gross phase lags suggests a coherent phenomenon where a combination of temperature and opacity rules the amplitude. While these variations have been attributed to $\mathrm{F}$ star pulsation, whether the overall brightness variations are due to pulsational hotspots on the $\mathrm{F}$ star and/or changes in the disk material density and opacity remains to be determined.

\subsubsection{Line Variations}

In addition to continuum variation largely due to obscuration of the F star by the opaque disk, there are numerous hydrogen absorption lines as previously noted, plus persistent $4.05 \mu \mathrm{m}$ $\operatorname{Br} \alpha$ emission (Backman et al. 1985). Eclipse-phased differences among these line strengths were noted as follows: $\mathrm{Br} \alpha$ remains in emission and essentially unchanged in overall strength (equivalent width) throughout the eclipse. In contrast, $2.16 \mu \mathrm{m} \mathrm{Br} \gamma$ absorption strength shows a decrease as the eclipse begins, although after mid-eclipse, the strength mimics $V$-band light variations (see Figure 3). This behavior is consistent with statements by Backman et al. and earlier researchers that the hydrogen does not clearly associate with either component in the $\epsilon$ Aurigae binary. Previously mentioned Spitzer IRS data also show hydrogen recombination emission, $\mathrm{Hu} \alpha$ at $12.4 \mu \mathrm{m}$

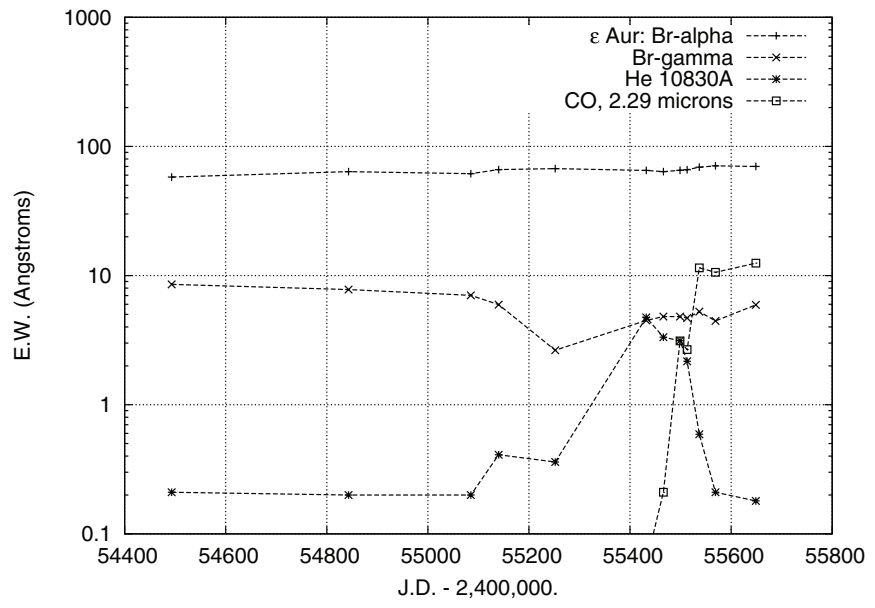

Figure 3. SpeX observations of equivalent widths of lines in $\epsilon$ Aurigae on selected dates. Data obtained on RJD 55,829 showed CO no longer present.

Table 2

SpeX + IRTF Monochromatic Fluxes $\left[\lambda f_{\lambda}\right.$ per $\left.10^{-10} \mathrm{~W} \mathrm{~m}{ }^{-2}\right]$ at Selected Continuum Wavelengths $(\mu \mathrm{m})$

\begin{tabular}{llccccccc}
\hline \hline JD & \multicolumn{1}{c}{ Date } & 1.1 & 1.65 & 2.1 & 3.15 & 3.95 & 4.64 & Notes \\
\hline $2,454,493$ & 2008 Jan 27 & 9.46 & 4.77 & 2.75 & 1.04 & 0.60 & 0.41 & Pre \\
$2,454,842$ & 2009 Jan 10 & $\ldots$ & $\ldots$ & 2.88 & 1.17 & 0.69 & $\ldots$ & \\
$2,455,084$ & 2009 Sep 10 & 7.77 & 3.97 & 2.31 & 0.81 & 0.46 & 0.31 & I \\
$2,455,140$ & 2009 Nov 4 & 6.63 & 3.38 & 1.95 & 0.74 & 0.40 & 0.30 & \\
$2,455,167$ & 2009 Dec 1 & 5.81 & 2.95 & 1.70 & 0.64 & 0.35 & 0.23 & \\
$2,455,250$ & 2010 Feb 24 & 4.18 & 2.11 & 1.25 & 0.46 & 0.26 & $\ldots$ & II \\
$2,455,432$ & 2010 Aug 24 & 4.79 & 2.45 & 1.40 & 0.50 & 0.27 & 0.20 & Mid \\
$2,455,467$ & 2010 Sep 27 & 5.37 & 2.75 & 1.61 & 0.62 & 0.33 & 0.25 & \\
$2,455,494$ & 2010 Oct 24 & 4.89 & 2.51 & 1.45 & 0.53 & 0.30 & 0.21 & \\
$2,455,499$ & 2010 Oct 29 & 4.57 & 2.22 & 1.27 & 0.34 & 0.17 & 0.12 & \\
$2,455,513$ & 2010 Nov 12 & $1.75:$ & $0.89:$ & $0.48:$ & $0.19:$ & $0.11:$ & $0.07:$ & cirrus \\
$2,455,537$ & 2010 Dec 6 & 4.91 & 2.48 & 1.46 & 0.58 & 0.32 & 0.21 & \\
$2,455,569$ & 2011 Jan 6 & 5.39 & 2.75 & 1.57 & 0.58 & 0.32 & 0.21 & \\
$2,455,649$ & 2011 Mar 29 & 12.5 & 6.08 & 3.47 & 1.26 & 0.71 & 0.44 & III \\
\hline
\end{tabular}

(Stencel 2007). Thus, it is reasonable to infer that the infrared spectrum would be dominated by nebular recombination lines, except for the dominance of the $\mathrm{F}$ star absorption spectrum when out of eclipse. We tested this idea by normalizing and differencing the pre-eclipse spectrum (2008 January 27) with the mid-eclipse spectrum (2010 August 24), both obtained with narrow slits. Line depths are less at mid-eclipse, and the scaled difference in the Brackett series is a clear set of weak emission lines (Figure 4). This is consistent with the reduced absorption equivalent width of $\mathrm{Br} \gamma$ (Figure 2(a)), and evidence for core emission reported in $0.65 \mu \mathrm{m} \mathrm{H} \alpha$ at mid-eclipse. This strongly suggests an extended low density distribution of ionized material between the components, the likes of which could be confirmed by interferometric spectroscopy (e.g., the VEGA instrument at CHARA Array-D. Mourard 2011, private communication) and/or high resolution mid-IR imaging.

\subsubsection{Appearance of the He I 10830 A Line at Mid-eclipse}

Unexpectedly, we witnessed the appearance of strong He I line absorption at $1.083 \mu \mathrm{m}$ in our closest to mid-eclipse spectrum (2010 August 24)—Figure 1(b). The extra absorption converted a $0.2 \AA$ equivalent width photospheric line prior to RJD 55,250 (possibly stellar He I, $250 \mathrm{m \AA}$ is consistent with normal B5V stars; Leone et al. 1995) into a feature as large as $5 \AA$ in equivalent width during/after mid-eclipse. The excess 


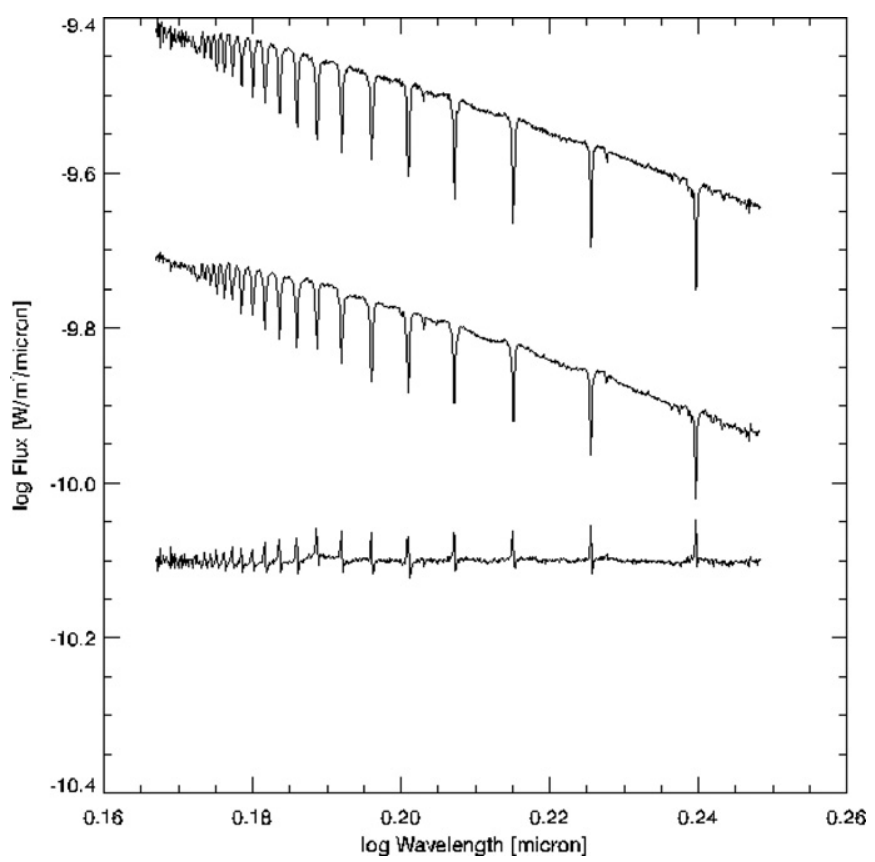

Figure 4. SpeX flux distribution and scaled differences of lines approaching the Brackett limit near $1.5 \mu \mathrm{m}$, pre-eclipse to mid-eclipse, showing the recombination spectrum of extended disk material in $\epsilon$ Aurigae.

absorption persisted for months thereafter [4.7 $\AA$ on RJD 55,432; $3.3 \AA$ on 55,$467 ; 3.1 \AA$ on 55,499 , and $2.2 \AA$ on 55,513 ], with the excess absorption finally disappearing in the 55,537 (2010 December 7) spectrum (0.6 $\mathrm{A}$ and $0.2 \AA$ on 55,569$)$. The velocity resolution with SpeX as used is only $70 \mathrm{~km} \mathrm{~s}^{-1}$, which is less than the anticipated motions in the $\epsilon$ Aurigae system $\left( \pm 30 \mathrm{~km} \mathrm{~s}^{-1}\right)$. Our observations are merely consistent with the absorption originating in either component (more likely the B star), as opposed to a high speed stream, for instance. Higher resolution spectra around the orbit could help secure the small but persistent absorption's point of origin.

The He I $1.083 \mu \mathrm{m}$ line arises from the ground state of the metastable triplet with components at $1.082909 \mu \mathrm{m}$, $1.083025 \mu \mathrm{m}$, and $1.083034 \mu \mathrm{m}$, in that order of laboratory intensity and from a lower level that is $19.82 \mathrm{eV}$ above ground. Thus, whether radiatively pumped or collisionally excited, He I $1.083 \mu \mathrm{m}$ represents plasma well in excess of $25,000 \mathrm{~K}$ and up to $230,000 \mathrm{~K}$ if in thermal equilibrium (Zarro \& Zirin 1986; Sanz-Forcada \& Dupree 2008). This heated region could represent the presence of a compact object, or more likely, accretion onto the star hidden inside the disk. The He II line at $1.0123 \mu \mathrm{m}$ was not detected, neither was the He I singlet at $2.06 \mu \mathrm{m}$, and the Ca II triplet near $0.85 \mu \mathrm{m}$ remained strong and unchanging. Ancillary lines that appeared briefly during this interval included $15950 \AA$ (Mg I) and $16896 \AA$ (Fe I?). As a side note, during the analysis of the He I $1.083 \mu \mathrm{m}$ line, we also note that $1.1289 \mu \mathrm{m} \mathrm{Pa}-\beta$ showed transient emission characteristics in the 2009 November 4 and 2009 December 1 spectra, and reports of transient emission in He I $0.6678 \mu \mathrm{m}$ (singlet) have been noted elsewhere.

The presence of He I during mid-eclipse provides constraints on the central star characteristics. We can estimate the gas density in the central part of the disk by noting the column density from CO (Hinkle \& Simon 1987): $3 \times 10^{20} \mathrm{~cm}^{-2}$, which implies $n_{\mathrm{H}}$ is $\sim 1.5 \times 10^{24} \mathrm{~cm}^{-2}$, and the latter is consistent with lack of soft X-rays per Wolk et al. (2010) who report that $n_{\mathrm{H}} \geqslant$
$1.25 \times 10^{24} \mathrm{~cm}^{-2}$. The column through the $3.8 \mathrm{AU}$ radius disk implies a number density approximately $2.6 \times 10^{10} \mathrm{~cm}^{-3}$, which is similar to the density deduced by KSM for the Hipparcos distance of $625 \mathrm{pc}$. Using the relative orbital speed reported in KSM and Hack \& Selvelli (1979), we deduce a He I zone width close to $1 \mathrm{AU}$. Then it is straightforward to compute the Stromgren sphere dimension for upper main-sequence stars, using tabulated mass, radius, and effective temperatures, and the He recombination rate of $1 \times 10^{-15} \mathrm{~cm}^{3} \mathrm{~s}^{-1}$ for $20,000 \mathrm{~K}$ and the number of ionizing photons shortward of $505 \AA$. We find that a B0V star can create a 1.3 AU Stromgren sphere, while a B5V star can manage only 0.05 AU. However, HHS and Howell et al. (2011) provided evidence that the central star more nearly resembles a B5V type photosphere, but with the superposition of UV nebular emission features. To achieve a larger Stromgren sphere with the B5V star, we can add the effect of accretion and boost the luminosity from accretion by $\mathrm{G} \mathrm{M}_{*}(d M / d t) / R_{*}$. With $d M / d t=1 \times 10^{-6} M_{\odot} \mathrm{yr}^{-1}(\sim 1$ Earth mass per year) the Stromgren sphere for the B5V star with accretion grows to $0.85 \mathrm{AU}$, close to the observed zone size, although the estimated thermal gain from accretion is $1 \times 10^{7} \mathrm{~K}$, and we assumed this results only in ionizing $505 \AA$ photons. A more detailed analysis is in preparation (see Pequette et al. 2011).

\subsubsection{Re-appearance of Molecular CO}

As indicated in Table 1, observations 32 days after nominal mid-eclipse (RJD 55,400) did not detect any CO (2-0) near $2.3 \mu \mathrm{m}$, while the next epoch, 67 days after mid-eclipse, did so. Figure 1(c) shows the re-appearance of CO (2-0) bands, which have strengthened in every subsequent observation, and can be anticipated to persist past the end of optical eclipse (4th contact). As noted by Takeuti (2011), the outer disk rotation period is approximately 3 years, assuming a 5.9 solar mass central star. In unperturbed Keplerian motion, the 35 day time interval noted here translates to $\sim 0.1$ AU or a remarkably small $1 \%$ of the disk circumference, over which $\mathrm{CO}$ is sublimated from, or radiatively driven away from, disk particles. For lack of more precise terminology, we will refer to this as a terminator shock where $\mathrm{F}$ star radiation is on the rise. The significance of the re-appearance of molecular $\mathrm{CO}$ lies in the gas-to-dust constraint it provides in understanding the disk structure and evolution. If $\mathrm{CO}$ is a result of comet-like sublimation of icy surfaces of solids in the disk, then searches at other wavelengths may succeed in detecting related molecules like $\mathrm{CO}_{2}, \mathrm{H}_{2} \mathrm{O}$, and $\mathrm{OH}$, for years after eclipse. While SpeX resolution was too low to resolve individual lines, the Gemini North IR Spectrometer (T. Geballe 2011, private communication) was used to obtain observations at high dispersion on 2011 January 4, and these confirmed the presence of $\mathrm{CO}(2-0)$ and (3-1) components at intensities comparable to those reported by Hinkle \& Simon (1987).

\subsection{Spitzer/IRAC}

IRAC (Fazio et al. 2004) on board the Spitzer Space Telescope (Werner et al. 2004) has, in warm mission phase, two operational channels that provide simultaneous 5.2 arcmin by 5.2 arcmin images at $3.6 \pm 0.3$ and $4.5 \pm 0.5 \mu \mathrm{m}$. The goal of this series of measurements was to provide a well calibrated but independent means of assessing the SED contribution of the cold disk relative to the $\mathrm{F}$ star continuum, and to seek evidence for disk temperature increase as post-eclipse phases begin to present themselves. A very clever strategy to avoid bright object 
Table 3

IRAC Observations

\begin{tabular}{|c|c|c|c|}
\hline $\begin{array}{l}\text { JD } \\
\text { Notes }\end{array}$ & Date & $\begin{array}{c}\text { Flux } \\
(\mathrm{Jy}) \\
(3.6 \mu \mathrm{m})\end{array}$ & $\begin{array}{c}\text { Flux } \\
(\mathrm{Jy}) \\
(4.5 \mu \mathrm{m})\end{array}$ \\
\hline $\begin{array}{l}2,454,947 \\
V=3.02\end{array}$ & 2009 Apr 26 & $66.3 \pm 0.05$ & $52.9 \pm 1.4$ \\
\hline $\begin{array}{l}2,455,283 \\
V=3.73\end{array}$ & 2010 Mar 28 & $42.3 \pm 0.6$ & $31.9 \pm 0.6$ \\
\hline $\begin{array}{l}2,455,305 \\
V=3.82\end{array}$ & 2010 Apr 19a & $42.3 \pm 0.2$ & $31.8 \pm 0.3$ \\
\hline $\begin{array}{l}2,455,305 \\
V=3.82\end{array}$ & 2010 Apr 19b & $42.5 \pm 0.2$ & $31.8 \pm 0.2$ \\
\hline $\begin{array}{l}2,455,500 \\
V=3.76\end{array}$ & 2010 Oct $30 a$ & $46.1 \pm 0.8$ & $33.6 \pm 0.2$ \\
\hline $\begin{array}{l}2,455,500 \\
V=3.76\end{array}$ & 2010 Oct $30 b$ & $46.4 \pm 0.8$ & $33.6 \pm 0.2$ \\
\hline $\begin{array}{l}2,455,523 \\
V=3.76\end{array}$ & 2010 Nov 22a & $45.5 \pm 0.6$ & $32.9 \pm 0.2$ \\
\hline $\begin{array}{l}2,455,523 \\
V=3.76\end{array}$ & 2010 Nov $22 b$ & $45.7 \pm 0.6$ & $32.9 \pm 0.2$ \\
\hline $\begin{array}{l}2,455,664 \\
V=3.40\end{array}$ & 2011 Apr 12 & $53.3 \pm 1.6$ & $41.2 \pm 1.1$ \\
\hline $\begin{array}{l}2,455,680 \\
V=3.35\end{array}$ & 2011 Apr 29 & $58.9 \pm 1.2$ & $45.9 \pm 0.2$ \\
\hline
\end{tabular}

saturation but retain photometric precision was invented by Steve Howell and was used to place the star image at the intersection of pixels. In this way we were able to obtain a series of photometric measurements during the semi-annual observing windows since early 2009, as detailed in Table 3. Donald Hoard re-did the photometric extractions using data processing and photometry procedures identical to that described in HHS. All uncertainties in the table below are random (scatter) only. They do not include the systematic uncertainty contributions.

We note that the IRAC continuum flux decline in ratio terms, between pre-eclipse (2009 April) and totality (2010 March), were [totality/pre-eclipse] at $3.6 \mu \mathrm{m}$ : 0.64 and at $4.5 \mu \mathrm{m}$ : 0.60, which are somewhat less than flux decline ratios reported by Backman et al. (1984), namely, at $L$ band: $0.52 \pm 0.06$ and at $M$ band: $0.54 \pm 0.07$. These are larger than the SpeX results comparing 2009 September and 2010 March fluxes, at $3.15 \mu \mathrm{m}$ : 0.54 and at $3.95 \mu \mathrm{m}$ : 0.56 . Calibration issues aside, we note that SpeX fluxes in 2009 January (when $V=3.00$ ) were $10 \%-15 \%$ higher than during 2008 January (when $V=2.99$ ). During 2009 April when the IRAC baseline was obtained, the star was brightening quickly, and this could account for the differences. Clearly, short-term variations must be monitored to determine changes among eclipse depths. In addition, spectral features intrinsic to the IRAC bands could play a role in these differences. We note that the in-eclipse variations are small, just a few percent, compared those of the optical light which are of order $10 \%$. However, the temporal coverage is too limited to make a strong statement.

The externally heated disk model (Takeuti 2011) suggests that as eclipse progresses, higher temperature facets of the disk ought to rotate into view. Comparing IRAC measurements before and after mid-eclipse, we find small increases of 4 and $2 \mathrm{Jy}$ in the respective channels. Further observations were obtained in 2011 spring to confirm this trend. During egress, $V$ band brightened by 0.4 mag (44\%) between 2010 November 22 and 2011 April 12. Meanwhile, IRAC fluxes rose by less: $7.7 \mathrm{Jy}(17 \%)$ and $8.3 \mathrm{Jy}$ (25\%), respectively, suggesting a second, uneclipsed source of flux is present at those wavelengths (the heated disk). The

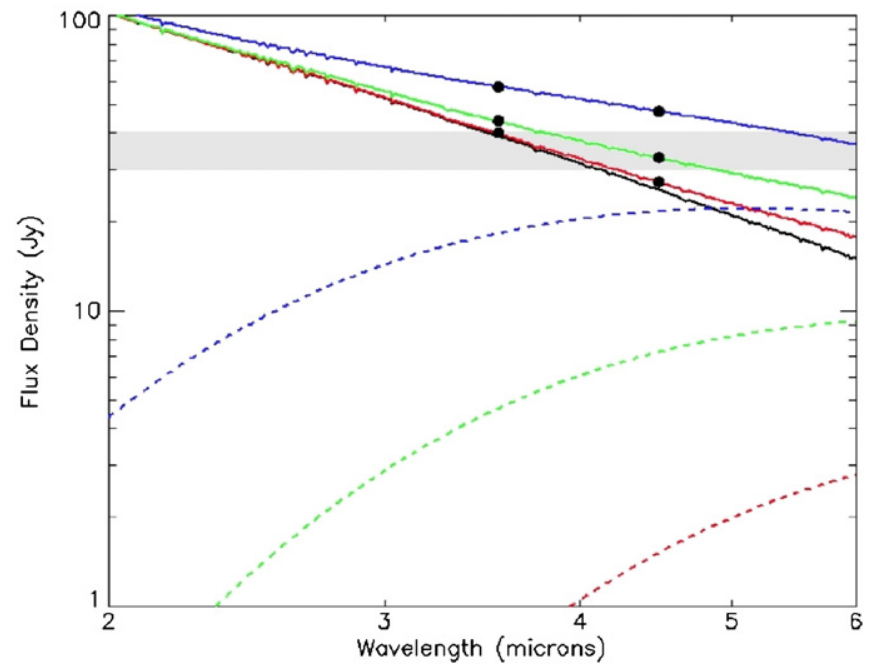

Figure 5. Plot of IRAC channels 1 and 2 flux predictions. The dashed lines show (from bottom to top) disk models (as in HHS10) with temperatures of $550 \mathrm{~K}$, $750 \mathrm{~K}$, and $1000 \mathrm{~K}$. The solid lines show (from bottom to top): F star template reduced by $50 \%$ to account for eclipse, and the sums of the eclipsed F star and the three disk models. See the text for details.

(A color version of this figure is available in the online journal.)

Table 4

BASS Observations

\begin{tabular}{lllr}
\hline \hline JD & \multicolumn{1}{c}{ Date } & Observers & $\lambda F_{11.6 \mu \mathrm{W} \mathrm{m}{ }^{-2}}$ \\
\hline $2,454,082$ & 2006 Dec 11 & MS/RR/DL & $3.774 \mathrm{E}-12$ \\
$2,454,713$ & 2008 Sep 3 & MS/RR/DL & $3.254 \mathrm{E}-12$ \\
$2,455,027$ & 2009 Jul 14 & MS/RR/DL & $3.381 \mathrm{E}-12$ \\
$2,455,165$ & 2009 Nov 29 & MS/RR/DL & $2.182 \mathrm{E}-12$ \\
$2,455,209$ & 2010 Feb 12 & MS/RR/DL & $1.834 \mathrm{E}-12$ \\
$2,455,260$ & 2010 Mar 4 & MS/RR/DL & $1.780 \mathrm{E}-12$ \\
$2,455,493$ & 2010 Oct 23 & MS/RR/DL & $1.844 \mathrm{E}-12$ \\
$2,455,769$ & 2011 Jul 29 & MS/RR/DL & $4.0 \mathrm{E}-12$ \\
\hline
\end{tabular}

2011 April 12 (29) data show recovery of flux from totality (2010 November), in the sense of flux ratios [egress/totality] at $3.6 \mu \mathrm{m}: 1.18(1.24)$ and at $4.5 \mu \mathrm{m}: 1.25(1.40)$, which is less change than the $V$ magnitude change (1.44).

Figure 5 plots IRAC channel 1 and 2 flux predictions. Preeclipse, we observed IRAC 1 and 2 flux densities of $66.2 \pm$ 3.0 and $52.9 \pm 2.4 \mathrm{Jy}$. Predictions from this simulation for heated disks during eclipse are 44.0 and $33.0 \mathrm{Jy}$ (750 K disk) and 57.3 and $47.3 \mathrm{Jy}$ (1000 K disk). Assuming non-systematic uncertainties smaller than 5\% (consistent with our first DDT observations), we have a sensitive means for diagnosing disk temperature variation during the balance of eclipse, when heated portions of the disk will rotate into view. The 2010 spring $(V=$ $3.82)$ and 2010 fall $(V=3.76)$ values seem to indicate a disk temperature closer to $700 \mathrm{~K}$ than the $550 \mathrm{~K}$ implied by preeclipse observations studied by HHS. A broader-band look at the mid-IR SED contributions is detailed below.

\subsection{BASS}

Additional observations were made with BASS, the Aerospace Corporation's Broadband Array Spectrograph System (Hanner et al. 1994), which spans the 2-14 $\mu \mathrm{m}$ wavelength region using two 58 element block impurity band linear arrays that simultaneously observe all spectral elements. Table 4 lists the observations made, with all of the observations conducted 


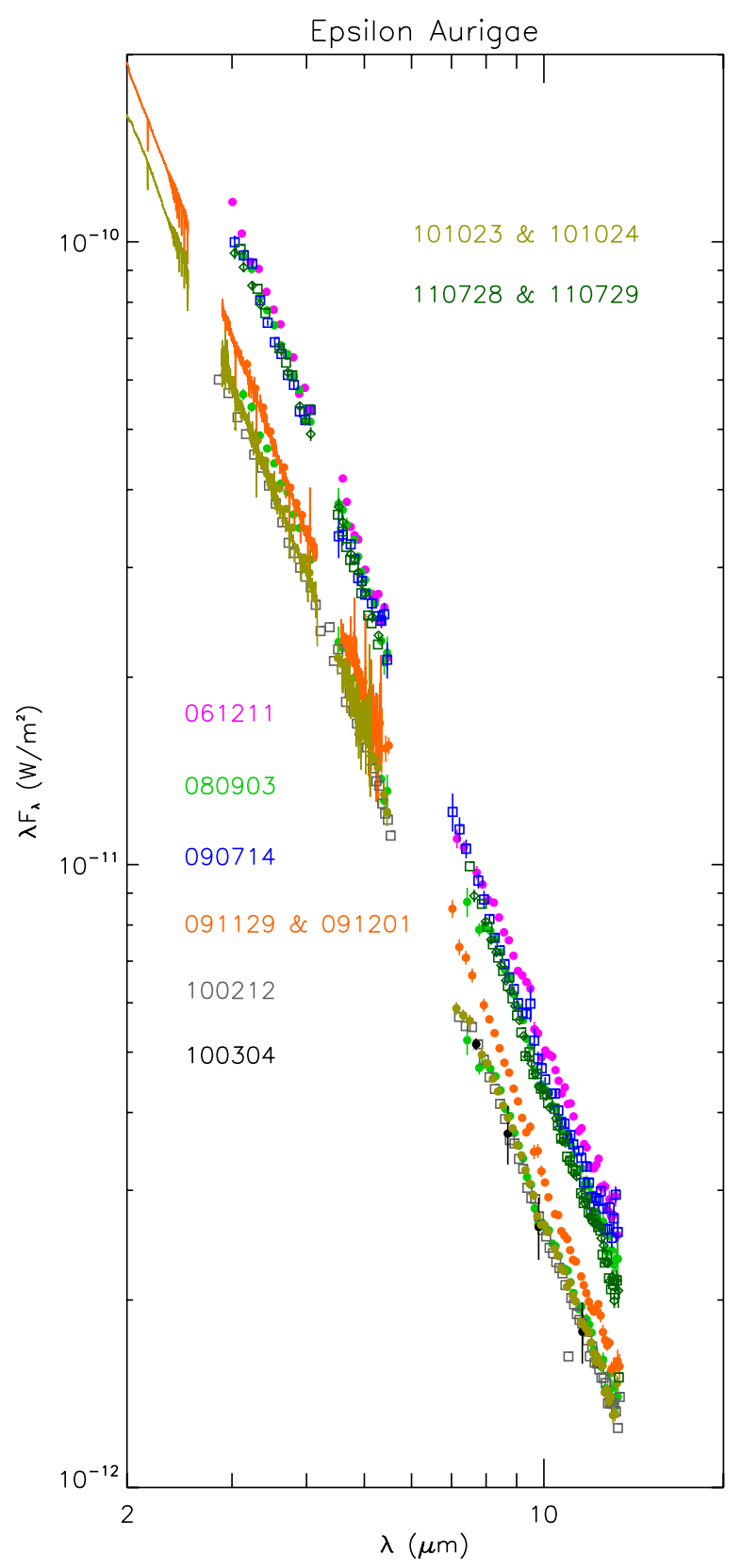

Figure 6. BASS observations of $\epsilon$ Aurigae on selected dates, 2006-2011.

at IRTF, except for 2010 February 12 which was conducted at AEOS atop Haleakela, HI. The calibrations and telluric corrections are similar to those described previously with SpeX, within $3 \%$ uncertainties.

A similar behavior in attenuation of the $F$ star continuum with wavelength is observed as eclipse progresses, with a flux ratio at $11.6 \mu \mathrm{m}$ of $1.90 \pm 0.07$ (2009 July 14 and 2010 March 4), which compares favorably to the $N$-band ratio of $1.75 \pm 0.09$ reported by Backman et al. (1984). During the course of BASS observations, little deviation from a blackbody continuum was observed, that is, no evidence for any strong silicate or carbonrelated solid state features at the usual mid-infrared wavelengths (Figure 6). However, the longest wavelengths do show departure from a $7500 \mathrm{~K}$ blackbody curve, consistent with the presence of a second source with a temperature of order $500 \mathrm{~K}$ or higher. Further observations are planned to confirm this trend.
Table 5

MIRSI + IRTF Observations

\begin{tabular}{lccc}
\hline \hline JD & Date & Observers & Notes \\
\hline $2,454,164$ & 2007 Mar 4 & GO/YPF & $10 \mu \mathrm{m}$ imaging \\
$2,454,484$ & 2008 Jan 17 & GO/YPF & $20 \mu \mathrm{m}$ imaging \\
$2,455,260$ & 2010 Mar 3 & MS/RR/DL & $10 \mu \mathrm{m}$ imaging \\
\hline
\end{tabular}

Table 6

MIRSI Observations, 2007 March 4, $V=3.04$

\begin{tabular}{lcccc}
\hline \hline$\lambda$ & $\epsilon$ Aur- $\alpha$ Aur & \pm & $\begin{array}{c}m \\
\epsilon \text { Aur })\end{array}$ & $\begin{array}{c}\epsilon \text { Aur } \\
(\mathrm{Jy})\end{array}$ \\
\hline 7.7 & 3.25 & 0.06 & 1.31 & 19 \\
9.8 & 3.37 & 0.05 & 1.44 & 11 \\
12.5 & 2.87 & 0.08 & 0.91 & 11 \\
\hline MIRSI & 2008 Jan 17 & $V=3.05$ & & \\
17.2 & 2.90 & 0.06 & 0.90 & 6 \\
17.9 & 2.81 & 0.05 & 0.80 & 6 \\
18.4 & 2.70 & 0.10 & 0.69 & 6 \\
20.8 & 2.99 & 0.03 & 0.96 & 4 \\
24.8 & 2.63 & 0.08 & 0.58 & 4 \\
\hline MIRSI & 2010 Mar 3 & $V=3.77$ & & \\
4.9 & 3.69 & 0.03 & 1.74 & 33 \\
7.7 & 3.62 & 0.04 & 1.68 & 14 \\
8.7 & 3.62 & 0.03 & 1.68 & 11 \\
9.8 & 3.60 & 0.03 & 1.67 & 9 \\
11.6 & 3.52 & 0.04 & 1.59 & 7 \\
12.5 & 3.44 & 0.06 & 1.51 & 7 \\
\hline
\end{tabular}

Table 7

MIRAC4 + MMT Observations

\begin{tabular}{llcl}
\hline \hline JD & Date & Observers & Notes \\
\hline $2,455,198$ & 2010 Jan 1 & JH/WH/AS & $V=3.67,10 \mu$ m silicates absent \\
$2,455,552$ & 2010 Dec 21 & RES/JLH/PH & $V=3.70,10 \mu$ m silicates absent
\end{tabular}

\subsection{MIRSI}

MIRSI is a mid-IR filter or CVF imager and low resolution spectrometer that operates in the 8-14 and $18-26 \mu \mathrm{m}$ bands and optimized for the IRTF (Kassis et al. 2008). Two epochs of pre-eclipse observations were obtained in 2007 and 2008, as described in Tables 5 and 6 , with Capella used as the primary comparison star. The $V$-band magnitudes reported for those pre-eclipse dates were 3.04 and 3.05, respectively. Adopted Capella monochromatic magnitudes and flux densities are $K=-1.83,3480 \mathrm{Jy} ; L=$ $-1.89,1420 \mathrm{Jy} ; M=-1.95,982 \mathrm{Jy} ; N=-1.93,235 \mathrm{Jy} ; Q=$ -2.03, 67 Jy (Persi et al. 1990; Tokunaga \& Vacca 2005). We will compare these observations with the Mid-Infrared Array Camera (MIRAC) and TNTCAM data in the next sections.

\subsection{MIRAC4}

MIRAC (Hoffmann et al. 1998) was originally built around a $128 \times 128 \mathrm{Si}$ :As $\mathrm{BIB}$ array that is sensitive to the $5-20 \mu \mathrm{m}$ region. The current configuration uses a $256 \times$ 256 array and is capable of both photometric imaging and low/moderate resolution spectroscopy. Observations were conducted at the Fred L. Whipple Observatory 6 meter MMT during 2010, as described in Tables 7 and 8. Capella was used as the primary comparison star. The photometric measurements will be compared with MIRSI and TNTCAM in the next section. The spectroscopic results indicate no significant changes in the mid-IR spectrum between early and later phases of totality, consistent with BASS results mentioned above. In both cases, the 
Table 8

MIRAC4 + MMT Observations, 2010 January 1, $V=3.67$, RJD 55198

\begin{tabular}{lcccc}
\hline \hline$\lambda$ & $\epsilon$ Aur- $\alpha$ Aur & \pm & $\begin{array}{c}m \\
(\epsilon \text { Aur })\end{array}$ & $\begin{array}{c}\epsilon \text { Aur } \\
(\mathrm{Jy})\end{array}$ \\
\hline 2.2 & 4.16 & 0.05 & 2.33 & $80 \pm 4$ \\
3.9 & 4.03 & 0.07 & 2.14 & $38 \pm 2$ \\
4.7 & 4.02 & 0.08 & 2.07 & $26 \pm 2$ \\
8.7 & $2.71:$ & 0.15 & $0.78:$ & $10:$ \\
9.8 & 3.39 & 0.06 & 1.46 & $8 \pm 1$ \\
10.5 & 3.10 & 0.11 & 1.17 & $9 \pm 1$ \\
11.8 & 2.77 & 0.01 & 0.82 & $6 \pm 1$ \\
12.5 & 2.43 & 0.03 & 0.48 & $6 \pm 1$ \\
\hline
\end{tabular}

Table 9

TNTCAM Observations

\begin{tabular}{llr}
\hline \hline JD & \multicolumn{1}{c}{ Date } & \multicolumn{1}{c}{ Notes } \\
\hline $2,450,026$ & 1995 Nov 5 & $11 \mu \mathrm{m}$ imaging, WIRO \\
$2,450,101$ & 1996 Jan 19 & 10, 11 $\mu \mathrm{m}$ imaging, WIRO \\
$2,450,145$ & 1996 Mar 3 & $10,11 \mu \mathrm{m}$ imaging, WIRO \\
$2,450,187$ & 1996 Apr 14 & $8-18 \mu \mathrm{m}$ imaging, MLOF \\
$2,450,333$ & 1996 Sep 7 & $5-18 \mu \mathrm{m}$ imaging, WIRO \\
$2,450,394$ & 1996 Nov 7 & $5-12 \mu \mathrm{m}$ imaging, WIRO \\
$2,450,426$ & 1996 Dec 9 & $5-18 \mu \mathrm{m}$ imaging, WIRO
\end{tabular}

continuum appears smooth and devoid of any strong $10 \mu \mathrm{m}$ silicate features at these phases.

\subsection{TNTCAM}

Denver University's TNTCAM was similarly constructed around a Rockwell $128 \times 128 \mathrm{Si}$ :As BIB array (Klebe et al. 1996) and was used for mid-infrared imaging at the Wyoming Infrared Observatory (WIRO, $2.4 \mathrm{~m}$ ) and the Mt. Lemmon Observing Facility (MLOF, 60 inch) during the 1990s, at a midpoint between the 1983 and 2010 primary eclipses_-suggested by Sheffer \& Lambert (1999) to be close to secondary minimum. A newer solution by Chadima et al. (2010) gives 2000 May as the time of secondary minimum. In either event, the decade of the 1990s should have presented the heated face of the disk toward Earth.

The observing teams at WIRO and MLOF included Denver astronomers Michelle Creech-Eakman, Mary Dahm, Matti Jalakas, Dimitri Klebe, Donald Neff, and Robert Stencel, plus support personnel from Wyoming (David Ciardi) and Mt. Lemmon (Drs. Mason and Williams). Observations obtained in those times are listed in Table 9. These secondary minimum observations occurred during a period when the $V$ band was reported to average $3.01 \pm 0.03$. Capella once again served as the primary comparison star, leading to the magnitudes and fluxes reported in Table 10. Analysis of the frames indicates that the mid-IR was $\sim 50 \%$ brighter during the mid-1990s than it was preceding the latest eclipse in 2007-2008 (MIRSI)—see Figure 7, indicative of the hotter face of the disk being oriented toward the observer during superior conjunction in the mid-1990s, in accordance with the model by Takeuti (1986, 2011). For completeness, we add published data obtained during this same timeframe, with the Midcourse Space Experiment (Egan \& Price 1996) and with ground-based JHKLM photometry reported by Taranova \& Shenavrin (2001). These observations show that the infrared spectrum of $\epsilon$ Aurigae was a factor of 2-3 brighter during secondary minimum, supportive of the model with an externally heated disk facing earth, as proposed by Takeuti $(1986,2011)$.
Table 10

Archival and TNTCAM Observations near Sec. Min., $V=3.02 \pm 0.03$

\begin{tabular}{|c|c|c|c|c|c|}
\hline Date & $\lambda$ & $\epsilon$ Aur $-\alpha$ Aur & \pm & $\begin{array}{c}m \\
(\epsilon \text { Aur })\end{array}$ & $\begin{array}{c}\epsilon \text { Aur } \\
(\mathrm{Jy})\end{array}$ \\
\hline $\begin{array}{l}\text { 1998-2000 } \\
\text { (Taranova \& } \\
\text { Shenavrin 2001) }\end{array}$ & $\begin{array}{c}1.25(\mathrm{~J}) \\
1.65(\mathrm{H}) \\
2.20(\mathrm{~K}) \\
3.75(\mathrm{~L}) \\
4.7(\mathrm{M})\end{array}$ & $\begin{array}{l}\cdots \\
\cdots \\
\cdots \\
\cdots \\
\cdots\end{array}$ & $\begin{array}{l}\cdots \\
\cdots \\
\cdots \\
\cdots \\
\cdots\end{array}$ & $\begin{array}{l}\cdots \\
\cdots \\
\cdots \\
\cdots \\
\cdots\end{array}$ & $\begin{aligned} 287 & \pm 8 \\
242 & \pm 11 \\
174 & \pm 5 \\
95 & \pm 3 \\
54 & \pm 1\end{aligned}$ \\
\hline $\begin{array}{l}M S X, 1999 \\
\text { (IRSA,) } \\
\text { Vizier) }\end{array}$ & $\begin{array}{r}4.29 \\
4.35 \\
8.28 \\
12.13 \\
13.5 \\
21.34\end{array}$ & $\begin{array}{l}\cdots \\
\cdots \\
\cdots \\
\cdots \\
\ldots \\
\ldots\end{array}$ & $\begin{array}{l}\cdots \\
\ldots \\
\cdots \\
\cdots \\
\cdots \\
\ldots\end{array}$ & $\begin{array}{l}\cdots \\
\ldots \\
\cdots \\
\cdots \\
\cdots \\
\ldots\end{array}$ & $\begin{array}{c}68 \\
72 \\
24 \\
12 \\
10 \\
5\end{array}$ \\
\hline $\begin{array}{l}1995 \text { Nov } \\
\text { WIRO }\end{array}$ & 11.6 & 2.96 & 0.28 & 1.01 & $13 \pm 1$ \\
\hline $\begin{array}{l}\text { 1996 Jan } \\
\text { WIRO }\end{array}$ & $\begin{array}{l}10.3 \\
10.3 \\
11.6 \\
11.6 \\
11.6\end{array}$ & $\begin{array}{l}2.95 \\
2.83 \\
2.73 \\
2.79 \\
2.73\end{array}$ & $\begin{array}{l}0.06 \\
0.06 \\
0.12 \\
0.10 \\
0.10\end{array}$ & $\begin{array}{l}1.02 \\
0.90 \\
0.78 \\
0.84 \\
0.78\end{array}$ & $\begin{array}{l}15 \pm 1 \\
17 \pm 1 \\
15 \pm 2 \\
14 \pm 1 \\
15 \pm 1\end{array}$ \\
\hline $\begin{array}{l}1996 \text { Mar } \\
\text { WIRO }\end{array}$ & $\begin{array}{l}10.3 \\
11.6\end{array}$ & $\begin{array}{l}2.74 \\
2.63\end{array}$ & $\begin{array}{l}0.13 \\
0.14\end{array}$ & $\begin{array}{l}0.81 \\
0.68\end{array}$ & $\begin{array}{l}19 \pm 2 \\
17 \pm 2\end{array}$ \\
\hline $\begin{array}{l}1996 \text { Apr } 22 \\
\text { MLOF* } \\
\text { Mason, } \\
\quad \text { Williams }\end{array}$ & $\begin{array}{r}2.2 \\
4.8 \\
7.8 \\
8.7 \\
9.8 \\
10.3 \\
11.6 \\
12.5\end{array}$ & $\begin{array}{l}3.20 \\
2.94 \\
2.80 \\
2.98 \\
2.86 \\
2.92 \\
2.63 \\
2.63\end{array}$ & $\begin{array}{l}0.05 \\
0.05 \\
0.08 \\
0.08 \\
0.08 \\
0.10 \\
0.10 \\
0.10\end{array}$ & $\begin{array}{l}1.37 \\
0.99 \\
0.86 \\
1.04 \\
0.93 \\
0.99 \\
0.70 \\
0.70\end{array}$ & $\begin{array}{c}193 \\
61 \\
29 \\
19 \\
17 \\
14 \\
16 \\
14\end{array}$ \\
\hline $\begin{array}{l}1996 \text { May } \\
\text { MLOF }\end{array}$ & $\begin{array}{r}7.8 \\
10.3 \\
11.6 \\
12.5\end{array}$ & $\begin{array}{l}2.90 \\
2.85 \\
2.71 \\
2.68\end{array}$ & $\begin{array}{l}0.22 \\
0.17 \\
0.18 \\
0.15\end{array}$ & $\begin{array}{l}0.96 \\
0.92 \\
0.76 \\
0.73\end{array}$ & $\begin{array}{l}26 \pm 5 \\
17 \pm 3 \\
16 \pm 3 \\
13 \pm 3\end{array}$ \\
\hline $\begin{array}{l}1996 \text { May } 29 \\
\text { MLOF* } \\
\text { Mason, } \\
\quad \text { Williams }\end{array}$ & $\begin{array}{r}2.2 \\
4.8 \\
7.8 \\
8.7 \\
9.8 \\
10.3 \\
11.6 \\
12.5\end{array}$ & $\begin{array}{l}3.32 \\
3.02 \\
2.91 \\
2.91 \\
2.80 \\
2.76 \\
2.52 \\
2.53\end{array}$ & $\begin{array}{l}0.05 \\
0.05 \\
0.08 \\
0.08 \\
0.08 \\
0.10 \\
0.10 \\
0.10\end{array}$ & $\begin{array}{l}1.49 \\
1.07 \\
0.97 \\
0.97 \\
0.87 \\
0.83 \\
0.59 \\
0.60\end{array}$ & $\begin{array}{r}172 \pm 9 \\
56 \pm 3 \\
27 \pm 2 \\
20 \pm 2 \\
18 \pm 1 \\
17 \pm 2 \\
17 \pm 2 \\
15 \pm 1\end{array}$ \\
\hline $\begin{array}{l}1996 \text { Sep } \\
\text { WIRO }\end{array}$ & $\begin{array}{r}4.6 \\
10.3 \\
11.6 \\
12.5\end{array}$ & $\begin{array}{l}2.98 \\
2.88 \\
2.79 \\
2.78\end{array}$ & $\begin{array}{l}0.02 \\
0.05 \\
0.06 \\
0.09\end{array}$ & $\begin{array}{l}1.03 \\
0.95 \\
0.84 \\
0.83\end{array}$ & $\begin{array}{l}57 \pm 1 \\
16 \pm 1 \\
14 \pm 1 \\
13 \pm 1\end{array}$ \\
\hline $\begin{array}{l}1996 \text { Nov } \\
\text { WIRO }\end{array}$ & $\begin{array}{r}4.6 \\
4.6 \\
7.8 \\
7.8 \\
10.3 \\
11.6\end{array}$ & $\begin{array}{l}3.05 \\
2.85 \\
2.86 \\
2.67 \\
2.79 \\
2.59\end{array}$ & $\begin{array}{l}0.02 \\
0.03 \\
0.19 \\
0.04 \\
0.06 \\
0.06\end{array}$ & $\begin{array}{l}1.10 \\
0.90 \\
0.92 \\
0.73 \\
0.86 \\
0.64\end{array}$ & $\begin{array}{l}56 \pm 2 \\
67 \pm 2 \\
27 \pm 5 \\
32 \pm 1 \\
18 \pm 1 \\
17 \pm 1\end{array}$ \\
\hline $\begin{array}{l}1996 \text { Dec } \\
\text { WIRO }\end{array}$ & $\begin{array}{r}4.6 \\
10.3 \\
11.6\end{array}$ & $\begin{array}{l}2.98 \\
2.61 \\
2.98\end{array}$ & $\begin{array}{l}0.07 \\
0.14 \\
0.51\end{array}$ & $\begin{array}{l}1.03 \\
0.68 \\
1.03\end{array}$ & $\begin{array}{l}57 \pm 4 \\
21 \pm 3 \\
12 \pm 6\end{array}$ \\
\hline
\end{tabular}

\section{RESULTS}

By means of a variety of infrared observations during the 2010 eclipse, we refined and solidified the following results and interpretations concerning the nature of $\epsilon$ Aurigae, some of 


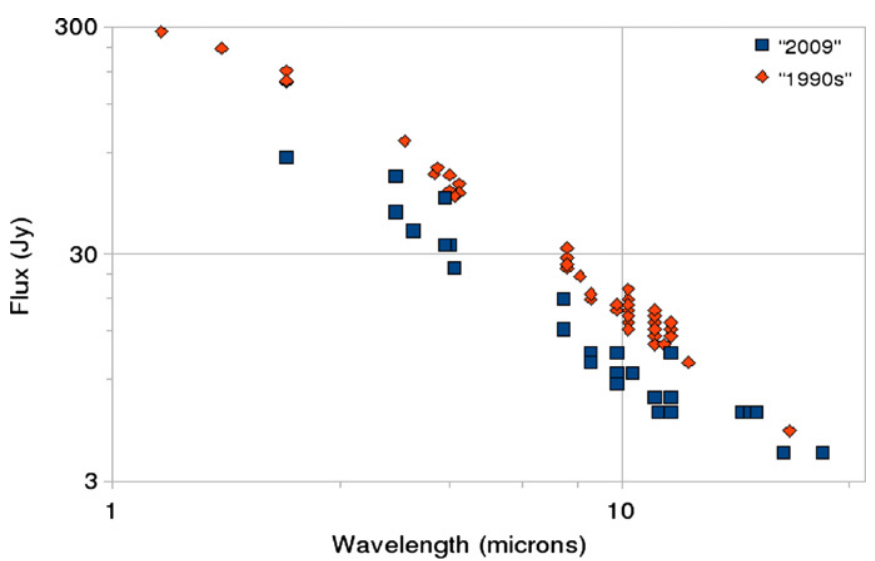

Figure 7. Mid-IR observations of $\epsilon$ Aurigae. The "2009" data refer to MIRSI photometry (obtained in 2007 and 2008, preceding the current eclipse) and IRAC photometry (pre-eclipse), with MIRAC data (obtained in early 2010, during totality). The "1995" data refer to a combination of TNTCAM photometry, along with $M S X$ and ground-based photometry published by Taranova \& Shenavrin (2001) obtained in 1996-1999, approximately during superior conjunction or secondary minimum. The comparison demonstrates that the disk was distinctly hotter during secondary minimum timeframe (1996) compared with total eclipse (2010).

(A color version of this figure is available in the online journal.)

which were introduced by researchers cited herein during the 1983 eclipse.

1. The IR eclipse depths during 2010 appear similar to that observed during the most recent (1983) eclipse (Backman et al. 1984), indicating relative stability of the objects involved over that timeframe. Spectral line profile changes indicate that the disk is embedded in a hydrogen recombination region (Hack \& Selvelli 1979).

2. Molecular $\mathrm{CO}$ absorption returned 57 days after nominal mid-eclipse (RJD 55,400), although it was not present at mid-eclipse plus 34 days narrowing the association with disk regions to a narrow terminator shock where external radiation from the $\mathrm{F}$ star begins to affect the disk (Hinkle \& Simon 1987; Takeuti 1986).

3. The newly found appearance of excess He I 10830A absorption associated with mid-eclipse and $\sim 90$ days thereafter is strongly suggestive of an accretion source embedded in the disk and consistent with a mid-B-main sequence object, as indicated by SED analysis (Hoard et al. 2010).

4. The lack of solid state features in BASS and MIRAC spectra, to date, suggests the dominance of micron-sized or larger particles in the disk, to account for the broadband opacities.

5. The comparison of pre- and in-eclipse data, along with superior and inferior conjunction data, appears to demonstrate differential heating of the disk due to the presence of the nearby $\mathrm{F}$ star, resulting in a $550 \mathrm{~K}$ surface facing the observer during primary eclipse and a $\sim 1100 \mathrm{~K}$ surface facing the observer during secondary minimum. Further mid- and far-IR observations can help constrain the heat capacity of the disk material, leading to more comprehensive disk models than presently available. Efforts should be made to image the disk out of eclipse with mid-IR interferometry during the coming decade, as the system approaches quadrature.

This work was supported in part by the bequest of William Herschel Womble in support of astronomy at the University of
Denver, by NSF grants AST 97-24506 and AST 10-16678, and JPL RSA 1414715 to the University of Denver, by NASA ADP grant NNX09AC73G to the University of Cincinnati, and by The Aerospace Corporation's Independent Research and Development Program. Observations reported here were obtained in part as Visiting Astronomers at the Infrared Telescope Facility, which is operated by the University of Hawaii under Cooperative Agreement NNX-08AE38A with the National Aeronautics and Space Administration, Science Mission Directorate, Planetary Astronomy Program. Observations reported here were obtained in part at the MMT Observatory, a joint facility of the Smithsonian Institution and the University of Arizona. Observations were also obtained in part at the University of Wyoming's Infrared Observatory (WIRO), involving access for which we are grateful. The first author thanks Dana Backman for the encouragement to obtain mid-IR data during the mid-1990s, and the many observers involved in those and all the data reported here. We also thank Jeff Hopkins, Brian McCandless, Thomas Rutherford, and the AAVSO for access to their visual observation records.

Facilities: IRTF (SpeX), MMT (MIRAC4), Spitzer (IRAC)

\section{REFERENCES}

Backman, D., Becklin, E., Cruikshank, D., et al. 1984, ApJ, 284, 799

Backman, D., \& Gillett, F. 1985, ApJ, 299, L99

Backman, D., Simon, T., \& Hinkle, K. 1985, PASP, 97, 1163

Chadima, P., Harmanec, P., Yang, S., et al. 2010, IBVS, 5937, 1

Cushing, M., Vacca, W., \& Rayner, J. 2004, PASP, 116, 362 (Spextool)

Egan, M., \& Price, S. 1996, AJ, 112, 2862

Fazio, G. G., Hora, J. L., Allen, L. E., et al. 2004, ApJS, 154, 10

Guinan, E., \& Dewarf, L. 2002, in ASP Conf. Ser. 279, Exotic Stars as Challenges to Evolution, ed. C. A. Tout \& W. van Hamme (San Francisco, CA: ASP), 121

Hack, M., \& Selvelli, P. 1979, A\&A, 75, 316

Hanner, M., Lynch, D., \& Russell, R. 1994, ApJ, 425, 274

Hinkle, K., \& Simon, T. 1987, ApJ, 315, 296

Hoard, D., Howell, S., \& Stencel, R. 2010, ApJ, 714, 549 (HHS)

Hoffmann, W. F., Hora, J. L., Fazio, G. G., Deutsch, L. K., \& Dayal, A. 1998, Proc. SPIE, 3354, 647

Hopkins, J., \& Stencel, R. 2011, AAS, 217, 257.01

Howell, S., Hoard, D., \& Stencel, R. 2011, AAS, 217, 257.07

Huang, S.-S. 1965, ApJ, 141, 976

Kassis, M., Adams, J., Hora, J., Deutsch, L., \& Tollestrup, E. 2008, PASP, 120 127

Klebe, D., Dahm, M., \& Stencel, R. 1996, in ASP Conf. Ser. 97, Polarimetry of the Interstellar Medium, ed. W. Roberge \& D. Whittet (San Francisco, CA: ASP), 79

Kloppenborg, B., Stencel, R., Monnier, J. D., et al. 2010, Nature (Lett.), 464, $870(\mathrm{KSM})$

Lambert, D., \& Sawyer, S. 1986, PASP, 98, 389

Leadbeater, R., \& Stencel, R. 2010, arXiv:1003.3617

Leone, F., Lanzafame, A. C., \& Pasquini, L. 1995, A\&A, 293, 457

Pequette, N., Stencel, R., \& Whitney, B. 2011, AAS, 218, 225.05

Persi, P., Ferrari-Toniolo, M., Ranieri, M., Marenzi, A., \& Shivanandan, K. 1990, A\&A, 237, 153

Rayner, J., Toomey, D., Onaka, P., et al. 2003, PASP, 115, 362

Sanz-Forcada, J., \& Dupree, A. 2008, A\&A, 488, 715

Sheffer, Y., \& Lambert, D. 1999, PASP, 111, 829

Stencel, R. 2007, in IAU Symp. 240, Binary Stars as Critical Tools \& Tests in Contemporary Astrophysics, ed. W. I. Hartkopf, E. F. Guinan, \& P. Harmanec (Cambridge: Cambridge Univ. Press), 202

Stencel, R. 2010, arXiv:1005.3738

Takeuti, M. 1986, Astrophys. Space Sci., 121, 127

Takeuti, M. 2011, PASJ, 63, 325

Taranova, O., \& Shenavrin, V. 2001, Astron. Lett., 27, 338

Tokunaga, A., \& Vacca, W. 2005, PASP, 117, 421

Vacca, W., Cushing, M., \& Rayner, J. 2003, PASP, 115, 389

Werner, M., Roellig, T. L., Low, F. J., et al. 2004, ApJS, 154, 1

Wolk, S., Pillitteri, I., Guinan, E., \& Stencel, R. 2010, AJ, 140, 595

Zarro, D., \& Zirin, H. 1986, ApJ, 304, 365 